Notice sur Bernardo Pasquini 1637-1710

Author(s): F. Boghen and M. L. Pereyra

Source: Bulletin de la Société française de musicologie, T. 2, No. 9 (Jul., 1921), pp. 189-191

Published by: Société Française de Musicologie

Stable URL: http://www.jstor.org/stable/924878

Accessed: 13-01-2016 12:41 UTC

Your use of the JSTOR archive indicates your acceptance of the Terms \& Conditions of Use, available at http://www.jstor.org/page/ info/about/policies/terms.jsp

JSTOR is a not-for-profit service that helps scholars, researchers, and students discover, use, and build upon a wide range of content in a trusted digital archive. We use information technology and tools to increase productivity and facilitate new forms of scholarship. For more information about JSTOR, please contact support@jstor.org. 


\section{NOTICE SUR BERNARDO PASQUINI}

$1637-1710$

C'est surtout comme grand organiste que l'histoire de la musique mentionne Bernardo Pasquini ; du compositeur, elle ne rappelle que les toccates, les cantates et quelques ouvrages moindres.

Ayant entrepris la publication de deux volumes, l'un de Fugues, l'autre de Toccates d'anciens maitres italiens ${ }^{1}$, je fus amené à approfondir les œuvres de ce compositeur si peu connu. Assurément il mérite de l'être davantage, à cause du caractère spécial de son style fait de grâce et de noblesse, vivace et sévère à la fois.

Déjà sa Toccala sur le Chant du Coucou (1698) est un des premiers exemples d'impressionisme musical : elle reproduit avec bonheur l'effet des harmonies de la nuit et celui plus étrange, du chant de l'oiseau. Elle peut heureusement soutenir la comparaison des pièces où Frescobaldi et Daquin ont tenté de rendre le charme de la voix du coucou au milieu du silence de la nature.

Les recherches auxquelles je me suis livré dans les catalogues des bibliothèques italiennes et étrangères, quoique non terminées, m'ont conduit à rédiger le catalogue que l'on verra plus loin; je serai reconnaissant à ceux qui voudront bien me communiquer tout renseignement relatif aux œuvres et à la vie de Bernardo Pasquini. Sa place est certainement parmi les musiciens les plus importants du

1. Fughe per Clavicembalo o Pianoforte di Antichi Maestri Ilaliani. Toccate per Clavicembalq o Pianoforte di Antichi Maestri Italiani (Ricordi, 1918). La maison d'édition "Musica " de Rome publiera prochainement un volume d'œuvres de Bernardo Pasquini du aux soins de MM. F. Boghen et A. Bonaventura. 
xvir siècle ; il a été le maître de Durante, de Gasperini et peut-être a-t-il lui-même fréquenté Scarlatti à Rome.

\section{CATALOGUE DES CEUVRES DE BERNARDO PASQUINI '}

\section{OEUVRES DRAMATIQUES}

Alcasta, B. E. - Alessandro aurante (incomplet), Br. M. - Arianna. B. E. - Artide (2 actes, 8905), B. C. P. - Caduta delle Amazzoni, B. E. Dov'è amore e pietà (1679), Br. M. - Eudosia (1692). U. B. W. - Idalina, B. N. - Idalina (3 actes, $\mathrm{n}^{\circ}$ 8906), B. C. P. - La donna ancora è fedele (1676), B. E. - La forza d'amore, F. W. M (copie; B. I. M. F.). - Lisinaco, B. E. - Seleuco (incomplet), Br. M. - Tirinto (3 actes, 1672), B. E.

\section{GINTATES}

Manuscrits : B. L. M.. A. S. P. - B. M. (466 et 467). - B. E., Dix-sept volumes (Airs Cantates, Opéras). - Aplauso musicale a 5 voci (D. 2359), B. I. M. F. - B. N. Un volume relié.

\section{ORATORIOS}

Il Martirio di S. Vito, H. B. W. - La sete di Cristo (composé entre 1687 et 1689), H. B. W, 18665. - Mosè, H. B. W. - S. Agnese (2 parties), B. E. - S. Alessio, H. B. W.

\section{AIRS DIVERS}

Canti per soprano e b c, B. M. - Codici Ashburnamiani, deux airs, B. L.

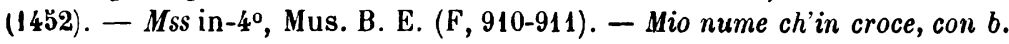
c., B. C. N. - Codici Barberini, B. V. (4148, già XLVII-19), 4149, 4153, 4157, 1458). - Harl. Mss, Br. M (1270 à 1273). - Add Mss. Br. M (5056). - Mio

1. Ce catalogue comprend les œuvres (mss.) connues jusqu'ici.

Abréviations du nom des Bibliothèques : B. V., Bibl. Vaticana, Rome. - B. E., Bibl. Estense, Modene. - B. L. M., Bibl. del Liceo Musicale, Bologne. - A. S. P., Archivio di S. Petronio, Bologne. - B. M., Bibl. Marciana, Venise. - B. L., Bibl. Laurenziana, Florence. - B. I. M. F., Bibl. Istituto musicale, Florence. B. C. N., Bibl. del Conservatorio, Naples. - B. N., Bibl. Nationale, Paris. - B. C. P., Bibl. du Conservatoire, Paris. - Br. M., British Museum, Londres. - F. W. M., Fitz William Museum, Cambridge. - H. B. W., Hofbibl, Vienne. - H. B. B., Hofbibl., Berlin. - K. M. D., Kön. Musiksammlung. Dresde. - U. B. W., Universitätsbibl., Wurzbourg. 
cor, mio cor, F. W. M. - Canzonette (n 8907, avec la mention : "Quasi tutto del Pasquini "), B. C. P.

\section{MUSIQUE DE CHAMBRE}

Un volume (Cantates, "Canzonette ", deux IIotets pour soprano et b. c,). B. E. - Add. Mss (trois volumes. Dates : 1703-1708), Br. M. (31501). Un volume, Br. M. (36661). - Duetti, Br. M. (5056). - Un volume (1702), Saggi di Contrappunto H. B. B. - Dialogo a 2 voci, K. M. D.

F. Boghen (Florence).

('Traduction de M. L. Pereryra 